\title{
OTIOTOMICS
}

Revista de economía, empresa y sociedad

Dossier "Claves para entender el turismo de hoy"

LOS EJES QUE CONDICIONAN EL DEBATE

\section{La investigación en el ámbito del turismo sostenible}

\section{Francesc González Reverté}

Profesor de los Estudios de Economía y Empresa (UOC)

RESUMEN Esta aportación pretende hacer un repaso de la investigación actual en turismo sostenible desde las dos vertientes. En primer lugar, se describen las claves del debate teórico que rodean la idea de sostenibilidad aplicada al sector turístico y, en segundo término, los ámbitos de investigación académico que son objeto del interés de la investigación en turismo y sostenibilidad. Esta aproximación permitirá elaborar una valoración global sobre los elementos que condicionan el debate en la sostenibilidad del turismo y los ejes temáticos preferentes donde se aborda esta cuestión.

PALABRAS CLAVE turismo; sostenibilidad; investigación; aproximaciones conceptuales

\section{Research in the field of sustainable tourism}

ABSTRACT This contribution seeks to provide an overview of current research into sustainable tourism from two angles. It describes firstly the key arguments in the theoretical discussion on the idea of sustainability in the tourism sector, and secondly the areas of academic research that are of interest for research into tourism and sustainability. This study will enable a global evaluation of not only the factors that influence the discussion on the sustainability of tourism, but also the central thematic areas in which this matter is addressed.

KEYWORDS tourism; sustainability; research; conceptual approaches 


\section{Introducción}

En la actualidad, la sostenibilidad aplicada al turismo es un ámbito de investigación plenamente consolidado. Desde la aparición, en los años noventa del siglo pasado, de postulados y planteamientos teóricos que apostaban por la sostenibilidad como paradigma intelectual, la investigación en turismo se ha dirigido en buena medida hacia este terreno. En un primer momento predominaba la discusión conceptual sobre los términos en que había que englobar los procesos de sostenibilidad y, poco a poco, se fueron introduciendo también aspectos pragmáticos y de aplicación práctica de la sostenibilidad a partir de casos de estudio (construcción de indicadores, cálculo de la capacidad de carga, medida de los impactos del turismo, herramientas para la gestión y planificación sostenible de destinos, etc.). A pesar de estos intentos de acercar la teoría sobre sostenibilidad a la práctica, una primera consideración sobre los límites de la investigación en turismo y sostenibilidad es precisamente el excesivo dominio de los debates teóricos por encima de las aportaciones aplicadas que resuelven problemas reales de los destinos y de la industria turística.

\section{El debate teórico sobre la sostenibilidad y su estatus paradigmático}

La noción de sostenibilidad ha sido un referente en el mundo del turismo durante las últimas décadas. La proliferación de grupos de investigación, publicaciones y manuales académicos, revistas especializadas, organización de congresos y debates, conferencias internacionales y foros de debate en Internet que han abordado esta temática da fe del interés social que suscita. Inicialmente, la sostenibilidad era un concepto nuevo que ampliaba y complementaba, apoyándose en un corpus teórico alternativo y con unos principios muy estructurados, la visión de la necesidad de cambiar modelos de funcionamiento en turismo, de introducir nuevos procesos de organización y funcionamiento en toda la cadena de valor y la consideración de que los gustos y las preferencias de la demanda obedecían a un cambio en la sensibilidad promovida por la introducción de valores éticos y el cambio social generacional. La irrupción del debate sobre la sostenibilidad como instrumento para un cambio de modelo generó la necesidad de justificar conceptualmente los argumentos teóricos que lo sostienen (equidad, aproximación holística, apuesta por el largo plazo y respeto por los principios de precaución). En este sentido, uno de los debates más importantes se ha situado alrededor de la (in)capacidad del turismo sostenible para seguir los principios del desarrollo sostenible teniendo en cuenta la tendencia inherente al crecimiento económico de este sector y la naturaleza dependiente de la lógica de producción del turismo a escala global (Sharpley, 2000). Este debate, iniciado hace veinte años, se fue estirando de forma que, si bien ha generado un corpus teórico completo capaz de cimentar los fundamentos de la sostenibilidad como paradigma científico, ha sido poco útil para proponer soluciones prácticas desde la sostenibilidad a los problemas reales de los espacios turísticos. Un efecto colateral del crecimiento paradigmático de la sostenibilidad ha sido el uso indiscriminado y gratuito del término en multitud de documentos y propuestas de desarrollo turístico. Las iniciativas del sector privado que caen en la categoría del greenwashing o las propuestas de desarrollo sostenible de destinos sin una estrategia política asociada son ejemplos de la consideración de la sostenibilidad como un paraguas de conceptos o, todavía peor, como una moda.

Este debate teórico inicial no es estéril, puesto que ha permitido centrar los elementos conceptuales para considerar aquello que es y que no es sostenible en turismo, pero ha generado cierta frustración en la medida en que ha retrasado la acción práctica hacia la introducción de los procesos de sostenibilidad en la industria turística. Actualmente, el debate ya no pasa tanto para reconocer hasta qué punto la sostenibilidad y el desarrollo son conceptos antagónicos, sino que se entiende que la sostenibilidad es un concepto flexible y que se tiene que adaptar a los diferentes contextos donde se quiere llevar a cabo. Es decir, a pesar de que hay que seguir los mismos principios y objetivos generales no se puede aplicar la misma estrategia de sostenibilidad en un contexto a pe- 
queña escala (por ejemplo, una comunidad rural que apuesta por el turismo endógeno con pocos turistas) que en un contexto donde la incidencia de la sostenibilidad es poliédrica y las afectaciones, problemas y repercusiones son mucho más complejos (por ejemplo, un destino de turismo de masas). Esta nueva visión no deja de ser una respuesta que alivia la carga conceptual en el debate y propone entender la sostenibilidad como un instrumento de trabajo práctico. La sostenibilidad se entiende como una herramienta de negociación (un horizonte divisado por los agentes que se ponen en contacto para debatir sobre el desarrollo del turismo en un lugar concreto) más que una norma absoluta (es decir, que acepta posicionamientos particulares derivados del contexto social y económico que vive un determinado destino o sector turístico).

La superación del debate paradigmático ha permitido centrar los esfuerzos hacia la generación de estudios aplicados. No obstante, existe todavía otro obstáculo importantísimo, puesto que no siempre se encuentra la suficiente voluntad política para llevar a cabo las propuestas de sostenibilidad alegadas. Por ejemplo, en el caso del turismo urbano sostenible, a pesar de que las administraciones locales y regionales incorporan la sostenibilidad en la agenda política hay pocas que integren en la práctica estos principios en las estrategias en los planes que guían el desarrollo del turismo en este ámbito (Maxim, 2016).

De hecho, los expertos consideran que se necesita dar paso a una visión mucho más global y amplia de los temas y retos del turismo sostenible si se quieren lograr resultados visibles. Por ejemplo, en un estudio realizado sobre publicaciones de investigación en la revista Journal of Sustainable Tourism entre el 2015 y el 2016, se constata que las contribuciones se centran sobre todo en aspectos de producto y consumidores, cambio climático, protección de los ambientes marítimos y costeros, protección de ecosistemas y biodiversidad, innovación y sostenibilidad, o la introducción de nuevas tecnologías para mejorar los resultados y la eficiencia en las pautas de producción, pero apenas abordan muchos de los temas que las Naciones Unidas consideran claves para conseguir un desarrollo sostenible, como, por ejemplo, la reducción de la pobreza, la erradicación del hambre, el bienestar de la población, la reducción de la desigualdad, los desequilibrios de género, el acceso a la energía sostenible, las ciudades sostenibles o la educación a lo largo de la vida (Bramwell y otros, 2017).

En el mismo estudio también se pone sobre la mesa el contraste existente entre la abundancia de aproximaciones especializadas y muy focalizadas (estudios de la sostenibilidad en festivales, museos, voluntariado, etc.) y la escasez de estudios que aborden temas desde una vertiente más amplia y holística, que permita llegar e interesar a grandes públicos. Se detecta, por lo tanto, una carencia de trabajos interdisciplinarios y con una óptica genérica y de enfoque global, mientras que predominan los estudios puntuales que solo explican parcialmente la complejidad de la sostenibilidad en el sistema turístico.

A esta carencia también hay que añadir tres reflexiones más sobre la manera en que se desarrolla la investigación en turismo sostenible. En primer lugar, existe un dominio de las aportaciones desde los países ricos, que a menudo responden a financiación pública o privada que condiciona el foco de interés, donde hay poca sensibilidad a la hora de recoger los problemas de las sociedades pobres; la abundancia de estudios de caso por encima de las comparativas entre diferentes casos de estudio, así como una carencia de aproximaciones basadas en métodos cualitativos y en la óptica longitudinal (Ruhanen y otros, 2015).

Finalmente, es preciso hacer mención de cuál es el marco general que condiciona la investigación en turismo y sostenibilidad. Esto es importante en la medida en que consiste en el marco de interés en el que se mueve la investigación. Es decir, según qué aproximaciones y preguntas se hacen, se define qué interesa y qué no, cómo deben abordarse las soluciones y qué políticas tienen que ir asociadas a la propuesta de acciones. En este sentido, la actividad de investigación en turismo y sostenibilidad se está dirigiendo hacia unas direcciones determinadas (Bramwell y otros, 2017):

1. Comprender mejor las actitudes y los comportamientos de los consumidores para poder influir sobre su toma de decisiones a la hora de hacer vacaciones para que sean más sostenibles.

2. Promover el cambio de las actividades y rutinas cotidianas de las personas para sensibilizarlas respecto a la sostenibilidad cuando actúan como turistas. Se trata, en definitiva, de promover comportamientos turísticos sostenibles cambiando previamente las relaciones de producción y consumo, las modas y las normas en la vida cotidiana. Es decir, se pretende aumentar el nivel de conciencia hacia la sostenibilidad de la sociedad en general para que cuando actúen se sea turista se exijan también unos parámetros y estándares similares. 
3. Estudiar nuevas pautas potenciales para la transformación social requerida para conseguir un turismo más sostenible. Se trata de ver cómo los recorridos históricos y culturales, las normas sociales preexistentes y las reglas institucionales previas de una sociedad o destino pueden condicionar la capacidad potencial para obtener mejores resultados de sostenibilidad en un futuro. Los conceptos de path-dependency y path-plasticity son elementos que expresan la existencia de unos acondicionamientos y rutinas culturales, institucionales y sociales que explican las decisiones finales presas por los consumidores que afectan a la sostenibilidad de sus vacaciones (por ejemplo, usar el coche en vez del tren a la hora de desplazarse por motivos de ocio).

4. El papel de la gobernanza como agente de refuerzo de la sostenibilidad en los destinos turísticos. Se considera que existe una conexión entre las maneras en que las sociedades organizan la regulación de sus sistemas políticos, normas y valores sociales y la voluntad y la capacidad de estas sociedades para introducir prácticas turísticas sostenibles.

5. La introducción de una perspectiva sistémica en turismo sostenible. En concreto, hay un interés para hacer investigaciones que se preocupan por las interconexiones entre los sistemas sociales y los naturales (feedbacks dinámicos, causalidad, conexión entre diferentes elementos, etc.) y la consideración de los criterios de vulnerabilidad y de resiliencia aplicados a los destinos turísticos.

6. Una visión crítica sobre el turismo y la sostenibilidad basada en la introducción de criterios éticos y políticos. Esta visión se interesa especialmente por las situaciones de desigualdad, explotación y opresión que puede generar el turismo, así como por la introducción de medidas de cambio y transformación de cariz social.

\section{Temas de interés en la investigación actual sobre sostenibilidad y turismo}

La propuesta de investigación en turismo y sostenibilidad en las principales revistas del sector es suficientemente amplia y heterogénea como para poderla repasar en tan breve espacio disponible. Por este motivo, y tomando como referencia las seis orientaciones de sostenibilidad anteriormente descritas, incluiremos alguna aportación temática relevante vinculada en cada una de ellas. Pero antes es ineludible explicar brevemente cómo y dónde ha evolucionado la investigación reciente en sostenibilidad y turismo. En términos generales, se puede decir que la tendencia inicial de generar debates teóricos ha ido dando paso a investigaciones más aplicadas, empíricas o evaluadoras de los impactos reales de la actividad y los espacios turísticos. La madurez de la investigación en turismo se percibe también en la elección de ámbitos de aplicación de las investigaciones sobre sostenibilidad, los cuales ya no solo se destinan a nichos turísticos, sino que se refieren a todo tipo de modalidades, incluido también el turismo de masas. Otra tendencia de la investigación en turismo y sostenibilidad es la ampliación de los temas y ámbitos de conocimiento, a pesar de que continúa habiendo unas áreas donde se concentra la investigación: espacios naturales y ecoturismo, impactos del turismo, medida y evaluación de la sostenibilidad, desarrollo, estudios de comportamiento y planificación de los espacios turísticos son los ámbitos donde se produce más investigación académica en turismo y sostenibilidad. No obstante, durante la última década se han incorporado con fuerza la investigación sobre turismo, el cambio climático y el calentamiento global. Por otro lado, empiezan a verse estudios de sostenibilidad en ámbitos de investigación en turismo relativamente poco habituales como los festivales (Stevenson, 2016), el pro-poor tourism (Hall, 2007), el ecofeminismo (Yudina, Bryan, Grimwood, 2016), el turismo urbano (Maxim, 2016), la gobernanza (Bramwell y Lane, 2011), el turismo de voluntarios (Wearing, 2001), los destinos turísticos inteligentes (Gretzel, 2011) o la filantropía (Novelli y otros, 2016) por poner solo algunos ejemplos, así como también aproximaciones desde varios métodos y paradigmas de investigación, como la grounded theory (Stumpf, Sandstrom, Swanger, 2016). 


\subsection{Comportamiento del consumidor}

Los temas destinados a comprender las actitudes y los comportamientos de los consumidores incluyen una parte importante de la investigación en turismo y sostenibilidad. En el trasfondo que justifica el interés por este ámbito de investigación encontramos el desnivel existente entre lo que los turistas declaran que harían y lo que realmente hacen en materia de sostenibilidad al viajar. La opinión de los turistas sobre aspectos como la compra de productos (por ejemplo, de turismo responsable y ambientalmente amigable), la voluntad de pagar más por un producto turístico sostenible o lo que declaran hacer en relación con su comportamiento real in situ presenta diferencias importantes. Se detecta, por lo tanto, un considerable margen de mejora para hacer aumentar el comportamiento responsable de los consumidores a la hora de hacer vacaciones. De hecho, los expertos consideran que los turistas son menos propensos que las empresas y los Gobiernos a introducir cambios para adquirir un estilo de vida más sostenible de vacaciones (tienden a reproducir las prácticas de ocio de su vida cotidiana) y a dar apoyo a productos turísticos sostenibles. Los temas de investigación que se abordan desde esta perspectiva son muy variados e incluyen aspectos sobre el conocimiento de los perfiles de la demanda (caracterización de los turistas en función de su posicionamiento ante la sostenibilidad), los factores que inciden en su comportamiento final (aspectos culturales y creencias, barreras personales, sesgo social, etc.), los aspectos de motivación en la elección de vacaciones y el comportamiento en el destino y los impactos ambientales asociados, las herramientas e instrumentos para reducir los comportamientos poco sostenibles mientras se hacen vacaciones (premios, etiquetas ecológicas, certificaciones, sensibilización y educación, campañas de comunicación, etc.). La preocupación por el calentamiento global incluye investigación cruzada sobre las emisiones de carbono por turismo. Se considera que no tiene solución si no se hace a partir de un cambio de comportamientos y que las transformaciones en las actitudes no son posibles de lograr de forma voluntaria, sino que hay que incentivarlas mediante políticas concretas (Eijgelaar y otros, 2016).

Entre los elementos de debate que se vinculan con la transformación del marco sociotecnológico como elemento de cambio hacia pautas de comportamiento y conducta cotidianas más sostenibles en el turismo hay también algunas aportaciones interesantes desde el campo de la movilidad o de las prácticas cotidianas (reciclaje, por ejemplo) aplicadas a la práctica turística. Un terreno todavía poco trabajado, pero que puede dar lugar, en un futuro cercano, a interesantes contribuciones es el de la economía colaborativa. Este movimiento permite abrir una dimensión ética en la forma en se adquieren los viajes de vacaciones. La economía colaborativa se asocia a una dimensión moral, y, en turismo, puede alinearse con proyectos alternativos que rechazan los supuestamente impersonales e injustos productos de turismo convencional. No obstante, existe una corriente crítica que reflexiona sobre la capacidad real de la economía colaborativa para generar auténticas alternativas que benefician a los más desfavorecidos y que denuncia el camuflaje de modelos empresariales convencionales bajo la bandera de estas prácticas (Dredge; Gyimóthy, 2015).

\subsection{Transformaciones sociales}

Entre los temas de investigación vinculados a las transformaciones sociales podemos destacar dos enfoques: la investigación sobre sostenibilidad social y el cambio social desde el punto de vista de la movilidad. El contexto del que parte la investigación sobre sostenibilidad social es la constatación de la necesidad de superar la óptica economicista en el análisis de la sostenibilidad. El interés por la dimensión no económica de la sostenibilidad permite incorporar nuevas perspectivas paradigmáticas y metodológicas (como las del turismo comunitario) y cambiar la óptica del análisis de los impactos, priorizando el enfoque de los efectos del turismo desde el punto de vista de la comunidad local. Los temas de interés que incluye esta orientación tienen que ver con los derechos humanos y el turismo, la igualdad de oportunidades, la distribución equitativa de los beneficios generados por el turismo, la reducción de la pobreza, el mantenimiento de las formas y medios de vida de la comunidad local ante la irrupción del turismo, la preservación de la cultura tradicional, la prevención ante la explotación laboral, la planificación turística comunitaria, la protección de recursos locales o la inclusión de la comunidad local en los procesos de participación. Uno de los temas centrales de investigación es, desde esta perspectiva, el análisis 
de la percepción de los efectos del turismo sobre los residentes y la comunidad local, y sus diferencias con la visión de otros agentes sociales. Es decir, interesa saber cuál es la actitud de los residentes ante los turistas y si esta visión es concordante o discordante con la de los otros actores. La aproximación conceptual a este tema es variada. A pesar de que una de las ópticas más presentes en las publicaciones parte de la teoría del intercambio social (donde se analizan los costes y beneficios que para cada actor social implica el desarrollo del turismo), también se ha abordado desde la perspectiva de la evolución de los destinos turísticos (ciclo de vida) (Diedrich, García-Buades, 2009) o a partir de nuevos enfoques conceptuales, como la solidaridad emocional y sus vínculos con el empoderamiento de los residentes (Woosnam, 2012).

\subsection{Movilidad y sosteniblidad}

La investigación sobre movilidad y sostenibilidad en turismo parte de la constatación de que el rápido ritmo de crecimiento de los viajes turísticos se relaciona con el calentamiento global y el cambio climático. Existe una preocupación entre el sector académico respecto a que la población parece poner por delante la voluntad de hacer vacaciones a generar cambios en la manera de hacerlas para reducir sus impactos ambientales. Es decir, se plantea que, entre los turistas, no existe voluntad para introducir cambios de comportamiento en la manera de moverse (reducir el número de viajes o viajar a destinos que se encuentren más cerca del lugar de origen) o de usar medios de transporte alternativos al avión o el automóvil. Algunos de los temas de investigación que surgen de esta aproximación tienen que ver con el análisis de los caminos de dependencia (path-dependence) como factor cultural que explica la continuidad en los comportamientos de movilidad de los turistas, la insuficiencia de las soluciones tecnológicas y la necesidad de transformar el sistema sociotecnológico para llegar a propuestas efectivas de sostenibilidad en el comportamiento de movilidad de los turistas o la búsqueda de un cambio estructural para dar respuesta política a la actual tendencia de viajar a bajo coste asociada a la «baja sostenibilidad». La actual tendencia de investigación en movilidad sostenible plantea, por lo tanto, un nuevo enfoque que intenta superar la visión del cambio de comportamiento del consumidor per se, para centrarse en la transformación de la estructura sociotecnológica que condiciona su comportamiento. No se trata de incidir sobre el cambio en el comportamiento de la demanda como público, sino de cambiar el comportamiento de quienes toman decisiones políticas, de la industria y del mundo de la investigación para que ejerzan su influencia social y política sobre la población. En resumen, la investigación en movilidad sostenible y turismo se centra en tres objetivos: 1) actuar sobre los factores del sistema sociotecnológico que condicionan el comportamiento de los turistas y de los responsables políticos; 2) desmitificar la tecnología como única solución a los problemas de movilidad sostenible; y 3) superar los tabúes en la toma de decisiones política.

\subsection{La gobernanza}

La gobernanza es otro de los puntos que centra el interés de investigación en el campo del turismo y la sostenibilidad. La insatisfacción que generan los sistemas de gobierno tradicionales que no incorporan elementos de participación social y de transparencia ha hecho surgir el interés por aquellos procesos de gobierno más efectivos y ajustados a contextos sociales específicos. Superar la rigidez de la burocracia jerárquica y redirigir el interés hacia el uso de redes más allá del Estado y de los mercados es uno de los criterios que hace que una gobernanza efectiva y justa sea un requisito imprescindible para lograr objetivos de sostenibilidad en turismo.

Los aspectos conceptuales sobre la gobernanza son recurrentes en las revistas académicas. Esto resulta crucial para la investigación, puesto que se plantea la óptica desde la cual se entiende la gobernanza por parte del mundo académico. Es decir, se plantea cuáles son los temas que vale la pena investigar, las recomendaciones y conclusiones por hacer y qué es la agenda futura. Se define la representación social sobre la gobernanza y aquello que afecta a la gestión del turismo y sobre cómo tienen que ser incorporados los residentes a la manera de gestionar los destinos. La descripción de tipologías de gobernanza también es un tema de investigación importante en este ámbito. Se trata de perfilar modelos que caracterizan formas de gobernanza en función de la forma 
de regulación que contienen (más flexibles o más jerarquizadas) y del equilibrio de poder que se establece entre el Estado y el resto de los actores sociales. Finalmente, también se detecta un interés por la dimensión temporal de la gobernanza y por las diferencias en la escala de análisis (global, regional o local); hay interés por las diferentes maneras en que se pueden abordar la gobernanza y los efectos que plantea según la escala territorial de referencia, y por los caminos de dependencia sociales y culturales que influyen sobre las condiciones de gobernanza en lugares concretos. El análisis temporal de la gobernanza hace hincapié en el aprendizaje social como pauta de gestión del destino. Desde esta perspectiva se analiza cómo los actores intercambian ideas, conocimientos y aspiraciones, y la coexistencia de visiones y planes de acción compartidos. Se entiende que la gobernanza se inserta en un proceso de aprendizaje continuo que permite reaccionar ante circunstancias cambiantes y aprender de las experiencias obtenidas (Gill; Williams, 2011).

\subsection{Cambio climático}

Entre los campos de investigación que se vinculan a la visión sistémica del turismo sostenible destaca el del cambio climático (Leyshon, 2014). Actualmente, existe una preocupación de fondo entre la comunidad científica internacional sobre la velocidad que toma el fenómeno y la manera en que afecta directamente el sector turístico. Por un lado, se evidencia que el cambio climático impacta sobre la actividad económica, el entorno ambiental y en los mismos humanos. Por otro lado, se constata también que el sector turístico no queda alejado de estos impactos, sino que, contrariamente, se ve afectado directamente tanto en lo que respecta al sector en conjunto como en destinos concretos (costeras, montaña, etc.) (Weaver, 2011). Finalmente, también es motivo de preocupación el hecho de que la industria turística esté contribuyendo directamente a la generación de gases de efecto invernadero y, por lo tanto, al calentamiento del planeta por culpa del incremento del transporte de viajeros (UNWTOUNEP-WMO, 2008). Dado que el factor climático es clave a la hora de hacer turismo y de elegir un destino (tiene que ver el confort percibido), puede convertirse en un elemento que redirija los flujos turísticos internacionales y que afecte a los sectores productivos locales (evidentemente al turismo, pero también a aquellos sectores de la economía con los que está directamente relacionado).

Los temas de investigación que se ponen actualmente sobre la mesa sobre la relación entre turismo y cambio climático son bastante diversos. Entre los más importantes se encuentran el desarrollo y la implementación de estrategias para frenar las condiciones que generan el cambio climático y tomar acciones preventivas, el cálculo de las emisiones procedentes del turismo y la creación de escenarios de futuro, la percepción de las preferencias climáticas de los turistas y sobre los impactos que el cambio climático genera en los destinos, las acciones de alivio de la pobreza vinculadas a la lucha contra el cambio climático en los países en vías de desarrollo, las respuestas adaptativas para tipos específicos de destinos y su vulnerabilidad, o los impactos del cambio climático sobre el comportamiento de la demanda y sus efectos sobre la estacionalidad.

\section{6. Ética y moral}

Finalmente, también hay un interés de investigación considerable por los aspectos éticos y morales del turismo (Butcher, 2003). Existe la idea de fondo de que los actores del turismo pueden desarrollar un sentido de la responsabilidad ética y moral que va más allá de la autocomplacencia en el viaje turístico y que pueden generar un cambio de comportamiento que acabe acercando al desarrollo sostenible. La idea de una filantropía del viaje turístico se discute en ámbitos de investigación concretos como el turismo comunitario, el turismo responsable o el turismo voluntario. En estos ámbitos se distinguen diferentes temas de investigación: 1) un foco de interés por el consumo turístico (cuál es el potencial de los consumidores responsables como agente de cambio social a través de sus decisiones de compra basadas en motivaciones altruistas); 2) un foco en la producción turística y en conocer cómo las creencias éticas influyen sobre las decisiones empresariales (qué barreras y límites hay hacia a la creación de productos a partir de empresas que se guían por la responsabilidad social corporativa, qué elementos éticos afectan a las decisiones de los agentes de la industria turística cuando crean y comercializan 
paquetes de producto turístico); 3) el tipo de relaciones éticas que se establecen entre los actores del turismo (entre consumidores y productores, como, por ejemplo, la creación de productos de estilo de vida para captar determinados segmentos de demanda; entre el sector turístico y la población local, como en el caso de los códigos de conducta para incidir sobre el comportamiento de los turistas en el destino; o la relación entre trabajadores y empresarios, como en el análisis de las condiciones laborales y salariales en el sector turístico); y 4) los aspectos políticos de la responsabilidad en turismo (como, por ejemplo, la discusión alrededor de cuál es la escala más adecuada para desarrollar efectivamente el turismo responsable, el debate sobre el papel de las ONGD como difusores o como engranaje de mercantilización del turismo de voluntariado y solidario, etc.).

\section{Una reflexión final}

La tendencia actual de la investigación en sostenibilidad y turismo parte de la detección de una serie de problemas reales que afectan a la industria turística (conciencia ética, formas de gestión más eficiente, maneras de reducir los impactos del cambio climático o de mejorar la movilidad, etc.). No obstante, los temas inducidos por estas preocupaciones todavía reposan principalmente sobre la discusión teórica y conceptual. Las aportaciones se orientan hacia los aspectos inherentes al desarrollo de la propia disciplina, a indicar pautas para establecer cambios en la estructura social y tecnológica de las colectividades, a aplicar la política turística de una manera más eficiente o a estudios aplicados a aportar soluciones sobre los impactos del turismo en destinos particulares o sectores empresariales. Es evidente que estas preocupaciones son legítimas y aportan contenido válido a la busca de instrumentos, políticas y acciones de sostenibilidad para la industria turística. No obstante, se echa de menos un discurso más cercano a las necesidades y preocupaciones de la población a la hora de plantear una forma diferente de hacer turismo. La percepción de una sostenibilidad social se manifiesta en cuatro preocupaciones de alcance general, las cuales probablemente aparecen más en los medios, blogs y espacios de opinión de las redes sociales que en artículos académicos, y que solo han recibido respuesta parcialmente desde la investigación en turismo y sostenibilidad. Tanto es así que probablemente se entienden más como una tendencia social que como preguntas de investigación que debe desarrollar la comunidad científica. Estos campos de interés social a los cuales habría que acercar la investigación futura son, desde nuestro punto de vista, los siguientes:

1. La noción de viaje profundo. Personalmente, más allá de la autenticidad, interesa saber qué necesitamos de las vacaciones y qué podemos devolver a cambio a los lugares que visitamos. ¿Cómo obtener una relación más personal y más significativa con los lugares que visitamos y cómo entender mejor las motivaciones personales que nos impulsan a hacer turismo?

2. El turismo geolocal. La aproximación local empieza a ser un aspecto central del viaje turístico. ¿Cómo disfrutar de experiencias turísticas de proximidad? ¿Las experiencias basadas en la alteridad y la distancia, que generan movilidad de largo recorrido, pueden desplazarse en función del reconocimiento de la proximidad y de la mejora de la interpretación de los lugares cotidianos?

3. El viaje turístico lento (slow trave). ¿Cómo hacer aumentar el interés por los viajes en transporte alternativos o a pie? ¿Cómo organizar el turismo de proximidad a través de un sistema de movilidad más sostenible? Son preguntas que pretenden encontrar una manera diferente de viajar y de entender la distancia no como una traba o un obstáculo por superar, sino como un aliado del goce de la experiencia turística.

4. Destinos con inteligencia territorial. Es necesario buscar fórmulas inteligentes, a partir de la tecnología, para que los destinos no desarrollen solo estrategias para incrementar el número de turistas, sino también para maximizar sus beneficios dirigiéndose preferentemente a aquellos segmentos que mejor correspondan a los contextos particulares y las necesidades concretas de cada lugar.

Empezar a dar respuesta a este tipo de preguntas permitirá continuar el camino comenzado que rehúye o reduce la discusión paradigmática y que progresivamente se va acercando y se va haciendo más sensible a las preocupaciones y necesidades expresadas desde la sociedad. 


\section{Referencias bibliográficas}

BRAMWELL, B. y otros (2017). «Twenty-five years of sustainable tourism and the Journal of Sustainable Tourism: looking back and moving forward». Journal of Sustainable Tourism. Vol. 25, n. 1, págs. 1-9.

BRAMWELL, B.; LANE, B. (2011). "Critical research on the governance of tourism and sustainability». Journal of Sustainable Tourism. Vol. 19, n. ${ }^{\circ}$ 4, págs. 411-421.

BUCKLEY, R. (2012). "Sustainable tourism: research and reality». Annals of Tourism Research. Vol. 39, n. 2, págs. 528-546.

BUTCHER, J. (2003). The moralisation of tourism. Sun, sand and... saving the world? Londres: Routledge.

COHEN, S. y otros (2016). «Finding effective pathways to sustainable mobility: bridging the science-policy gap». Journal of Sustainable Tourism. Vol. 24, n. ${ }^{\circ}$ 3, págs. 317-334.

DIEDRIG, A.; GARCIA-BUADES, E. (2009). «Local perceptions of tourism as indicators of decline». Tourism Management. Vol. 30, n. ${ }^{\circ}$, págs. 512-521.

DREDGE, D.; GYMÓTHY, S. (2015). «The collaborative economy and tourism: critical perspectives, questionable claims and silenced voices». Tourism Recreation Research. Vol. 40, n. 3, págs. 286-302.

EIJGELAAR, E. y otros (2016). "Consumer attitudes and preferences on holiday carbon footprint information in the Netherlands». Journal of Sustainable Tourism. Vol. 24, n. ${ }^{\circ}$ 3, págs. 398-411.

GILL, A.; WILLIAMS, P. (2011). «Rethinking ressort growth: understanding evolving governance strategies in Whistler, British Columbia». Journal of Sustainable Tourism. Vol. 19, n. 4-5, págs. 629-648.

GRETZEL, U. (2011). «Intelligent systems in tourism. A social science perspective». Annals of Tourism Research. Vol. 38, n. ${ }^{\circ}$ 3, págs. 757-779.

HALL, M. (ed.) (2007). Pro-poor Tourism: who benefits? Clevedon: Channel View.

LEYSHON, C. (2014). "Critical issues in social science climate change research». Contemporary Social Science: Journal of the Academy of Social Science. Vol. 9, n. ${ }^{\circ} 4$, págs. 359-373.

LU, J.; NEPAL, S. (2009). «Sustainable tourism research: an analysis of papers published in the Journal of Sustainable Tourism». Journal of Sustainable Tourism. Vol. 17, n. ${ }^{\circ}$, págs. 5-16.

MAXIM, C. (2016). «Sustainable tourism implementation in urban areas: a case study of London» Journal of Sustainable Tourism. Vol. 24, n. ${ }^{\circ}$ 7, págs. 971-989.

NOVELLI, M. y otros (2016). «Travel philanthropy and sustainable development: the case of the Plymouth-Banjul Challenge». Journal of Sustainable Tourism. Vol. 24, n. 6, págs. 824-845.

RUHANEN, L. y otros (2015). "Trends and patterns in sustainable tourism research: a 25-year bibliometric analysis». Journal of Sustainable Tourism. Vol. 23, n. 4, págs. 517-535.

SHARPLEY, R. (2000). «Tourism and sustainable development: exploring the theoretical divide». Journal of Sustainable Tourism. Vol. 8, n. ${ }^{\circ}$, págs. 1-19.

STEVENSON, N. (2016). «Local festivals, social capital and sustainable destination development: experiences in East London». Journal of Sustainable Tourism. Vol. 24, n. ${ }^{\circ}$ 7, págs. 990-1006.

STUMPF, T.S.; SANDSTROM, J.; SWANGER, N. (2016). «Bridging the gap: grounded theory method, theory development, and sustainable tourism research". Journal of Sustainable Tourism. Vol. 24, n. ${ }^{\circ} 12$, págs. 1691-1708.

UNWTO-UNEP-WMO (2008). Climate change and tourism. Responding to global challenges. Madrid: UNWTOUNEP.

WEAVER, D. (2011). «Can sustainable tourism survive climate change?». Journal of Sustainable Tourism. Vol. 19, n. ${ }^{\circ} 1$, págs. 5-15.

WEARING, S. (2001). Volunteer tourism: experiences that make a difference. Oxon, UK: CABI Publishing.

WOOSNAM, K.M. (2012). «Using emotional solidarity to explain resident's attitudes about tourism and tourism development». Journal of Travel Research. Vol. 51, n. 3, págs. 315-327.

YUDINA, O; BRYAN, S.; GRIMWOOD, R. (2016). «Situating the wildlife espectacle: ecofeminism, representation and polar bear tourism». Journal of Sustainable Tourism. Vol. 24, n. ${ }^{\circ}$ 5, págs. 715-734.

ZHANG, H. y otros (2017). "Creating a scale for assessing socially sustainable tourism». Journal of Sustainable Tourism. Vol. 25, n. ${ }^{\circ}$, págs. 61-78. 


\section{Francesc González Reverté}

fgonzalezre@uoc.edu

Profesor de los Estudios de Economía y Empresa (UOC)

Doctor en Geografía por la UAB y profesor de los Estudios de Economía y Empresa de la UOC. Es profesor del grado de Turismo y del máster de Turismo, sostenibilidad y nuevas tecnologías. Coordina el grupo de investigación Laboratorio del Nuevo Turismo de la UOC. Sus líneas de investigación principales se centran en el campo del turismo de sol y playa, los eventos, la relación entre la tecnología y el turismo y la sostenibilidad en turismo.

Los textos publicados en esta revista están sujetas -salvo que se indique el contrario- a una licencia de Reconocimiento 3.0 España de Creative Commons. Podéis copiarlos, distribuirlos, comunicarlos públicamente y hacer obras derivadas siempre que reconozcáis los créditos de las obras (autoría, nombre de la revista, institución editora) de la manera especificada por los autores o por la revista. La licencia completa se puede consultar en http://creativecommons.org/licenses/by/3.0/es/deed.ca.

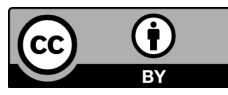

